\title{
Modeling of Unsteady Aerodynamic Loads
}

\author{
A. Da Ronch, ${ }^{*}$ A. McCracken, ${ }^{\dagger}$ K. J. Badcock ${ }^{\ddagger}$ \\ School of Engineering, University of Liverpool, Liverpool, United Kingdom \\ M. Ghoreyshi, § and R. M. Cummings ฯ \\ Modeling and Simulation Research Center, USAF Academy, CO, USA
}

\begin{abstract}
Time-accurate solutions of the Euler or Navier-Stokes equations are still nowadays a computationally expensive approach for applications where a magnitude of parameters has to be investigated. This work focuses on flight dynamics-related studies. The generation of several low-order models for the evaluation of unsteady and non-linear aerodynamic loads are investigated. The validity of low-order models presented is assessed by comparing the model output with unsteady time-accurate Computational Fluid-Dynamics (CFD) simulations. The test case is the NACA 0012 airfoil. The low-order models considered are: a non-linear model based on aerodynamic derivatives, a Volterra model, a surrogate-based recurrence-framework model, linear indicial functions and radial basis functions trained with neural networks.
\end{abstract}

\section{Nomenclature}

$c \quad=$ mean aerodynamic chord

$C_{m} \quad=$ pitching moment coefficient

$C_{m_{q}}+C_{m_{\dot{\alpha}}} \quad=$ pitching moment damping coefficient

$f \quad=$ dimensional frequency

$k \quad=$ reduced oscillation frequency $\left(k=\omega c /\left(2 U_{\infty}\right)\right)$

$M \quad=$ freestream Mach number

$N \quad=$ number of time steps in a CFD solution

$N_{T} \quad$ number of training CFD solutions

$q \quad=$ angular rate

$\mathbf{R} \quad=$ residual in the $\mathrm{CFD}$ equations

Re $\quad=$ Reynolds number $\left(R e=U_{\infty} c / \nu\right)$

$t \quad=$ physical time

$t^{*} \quad=$ non-dimensional time $\left(t^{*}=t U_{\infty} / c\right)$

$U_{\infty} \quad=$ freestream speed

$\mathbf{W} \quad=$ conservative variables in the CFD equations

$\underline{x} \quad=$ relevant external inputs for pitching case

$y \quad=$ any longitudinal aerodynamic loads in low-order model equations

Greek

$\alpha \quad=$ angle of attack

$\alpha_{0} \quad=$ mean angle of attack

$\alpha_{A} \quad=$ amplitude of oscillatory motion

$\nu \quad=$ kinematic viscosity of air

$\omega \quad=$ oscillation frequency $(\omega=2 \pi f)$

$\Phi, \Psi \quad$ = function which maps inputs to outputs in low-order model equations

\footnotetext{
*PhD Student, AIAA Member, Corresponding Author. Tel.: +44(0)151-7948035, Email: A.Da-Ronch@liverpool.ac.uk $\dagger$ PhD Student

$\ddagger$ Professor, AIAA Senior Member

$\S$ Research Fellow, AIAA Senior Member

TProfessor of Aeronautics, AIAA Associate Fellow
} 


\section{Introduction}

Determining the stability and control characteristics of aircraft at the edge of the envelope is one of the most difficult and expensive aspects of the aircraft development process. Non-linearities and unsteadiness in the flow are associated with shock waves, separation, vortices and their mutual interaction, which can lead to uncommanded motion and uncontrollable departure. If these issues are discovered at flight test, the aircraft development can suffer significant delays, a rise in production costs and detrimental effects on performance. There have been numerous examples of aircraft experiencing uncommanded activity, as reported, for instance, in. ${ }^{1}$ Following an extensive resolution process, immediate improvements are typically achieved by minor configuration changes and modifications to the flight control system and control augmentation laws. To provide a better fundamental understanding of the flow physics causing degraded characteristics, computational approaches have been used. ${ }^{2}$ The development of a reliable computational tool for prediction of these important issues would allow the designer to screen different configurations prior to building the first prototype, reducing overall costs and limiting risks. ${ }^{3}$

There are three traditional approaches to determine the stability and control characteristics. Flight testing the actual aircraft is the most accurate but also the most expensive of these methods. ${ }^{4}$ With a finalized configuration, the aircraft has to be operational and the required time to complete a flight test program can vary from several months to years. Wind-tunnel testing of scaled models is cheaper than flight testing yet providing accurate measurements. However, blockage, scaling and Reynolds number effects together with support interference issues prevent the proper modelling of the full scale aircraft behaviour. ${ }^{5}$ The final method comprises a combination of data sheets, linear aerodynamic theory and empirical relations. ${ }^{6}$ Due to its simplicity, this method has received widespread use for predicting aerodynamic derivatives in the conceptual design. However, the use of this approach is suspect when extended to novel aircraft configurations and to flight conditions dominated by non-linear aerodynamic effects.

A reasonable compromise between testing procedures and semi-empirical approaches is Computational Fluid Dynamics (CFD), which represents the state of the art in modelling non-linear flow physics. CFD solvers have reached a level of robustness and maturity to allow routine use on relatively inexpensive computer clusters. These techniques have successfully modelled the non-linear aerodynamic behaviour of aircraft at full scale Reynolds numbers, ${ }^{7}$ and investigated the transonic regime which is the most critical speed range for aircraft instabilities. However, the high fidelity realized in a CFD simulation comes with an additional cost in the execution time, which makes it not suitable for a variety of applications.

With regard to stability and control analysis, forces and moments dependencies on flight and control states are expressed in tabular form. A reasonable aerodynamic database to cover the expected flight envelope can easily contain up to about hundred-thousand or even million entries. If CFD is the source of the data and a "brute force" approach considered to filling the aerodynamic database, this would be prohibitely expensive even with the access to modern supercomputing facilities. When confronted to viscous CFD calculations of a three-dimensional geometry, an estimated time of 158 years was suggested for populating a database. ${ }^{8}$ An alternative approach was addressed in a previous study based on sampling, reconstruction and data fusion of variable-fidelity aerodynamic data. ${ }^{9}$ For a completely new design, it was found that tables can be generated with about hundred steady-state calculations, and on the order of ten solves when the already existing aerodynamic database for a given configuration needs to be updated for a new incremented geometry. ${ }^{10}$ This is most likely the scenario realized in the iterative process of the aircraft design and in the study of novel configurations. ${ }^{11,12}$

Whilst the prediction of static stability derivatives can be done with present off-the-shelf CFD tools, computation of dynamic derivatives using CFD requires the ability to compute the aerodynamic response to time-dependent prescribed motions. ${ }^{13,14}$ Typically, a time-accurate CFD analysis employs a dual-time stepping scheme, and a steady state is marched in pseudo time at each physical time step. Decay of initial transients in the aerodynamic response can require simulation of several oscillatory cycles, and the computational mesh is updated at each physical time step to conform to the applied motion. Then, postprocessing of the time domain data becomes necessary to extract dynamic derivatives. Roughly, a timeaccurate solution is as expensive as on the order of hundred steady calculations. While tolerable for a single point condition, this type of simulation is too expensive when compared to the magnitude of parameters to be investigated. An alternative to time-marching is offered by spectral methods. The harmonic balance method, which approximates the non-linear problem using a number of complex Fourier modes, was implemented and tested in the transonic speed range for a number of two- and three-dimensional configurations. ${ }^{15}$ While retaining the fidelity of a non-linear CFD solution to a high degree, a significant reduction in computational 
time was observed. ${ }^{16-18}$

Previous works ${ }^{19,20}$ substantiated the limitations of tabular models when comparing to CFD and flight tests data, and this motivates the current study. The overall objective is to put in place a framework of different low-order models for representation of non-linear unsteady aerodynamic loads. In this view, CFD is combined with predictive modelling techniques of low complexity to allow efficient and accurate predictions of the stability and control characteristics prior to the costly phases of wind tunnel and flight testing. The question whether the traditional model based on the concept of aerodynamic derivatives retains sufficient accuracy in predicting unsteady non-linear phenomena to pay off the significant initial cost incurred generating the aerodynamic database will be assessed.

The article continues with a description of the CFD solvers used in the cases presented. Several aerodynamic models are reviewed, and results for a two-dimensional test case are finally introduced.

\section{CFD Solver Formulation}

\section{A. PMB (University of Liverpool)}

The flow solver used at the University of Liverpool is the Parallel Multi-Block (PMB) solver. PMB is a well-established research code which solves the Euler and Reynolds-Averaged Navier-Stokes (RANS) models on block structured grids using an unfactored implicit method. The equations are discretised on curvilinear multi-block body conforming grids using a cell-centred finite volume method which converts the partial differential equations into a set of ordinary differential equations. A wide variety of unsteady flow problems, including aeroelasticity, cavity flows, aerospike flows, delta wing aerodynamics, rotorcraft problems and transonic buffet have been studied using this code. Validation studies against flight data for the F-16XL aircraft, ${ }^{7}$ a delta wing experiencing shock-induced vortex breakdown ${ }^{21}$ and multiple vortex flow on an unmanned combat air vehicle model ${ }^{22}$ have been conducted with this code. The main features of the CFD solver are detailed in Badcock et al. ${ }^{23}$

A fully implicit steady solution of the Euler or RANS equations is obtained by advancing the solution forward in time by solving the discrete nonlinear system of equations

$$
\frac{\mathbf{W}^{n+1}-\mathbf{W}^{n}}{\Delta t^{*}}=-\mathbf{R}\left(\mathbf{W}^{n+1}\right)
$$

The term on the right hand side, called the residual, is the discretisation of the convective terms, given here by Osher's approximate Riemann solver, ${ }^{24}$ MUSCL interpolation ${ }^{25}$ and Van Albada's limiter. Equation (1) is a nonlinear system of algebraic equations which is solved by an implicit method, the main features of which are an approximate linearisation to reduce the size and condition number of the linear system, and the use of a preconditioned Krylov subspace method to calculate the updates.

The steady state solver is applied to unsteady problems within a pseudo time stepping iteration ${ }^{26}$ which at each real time step is written as

$$
\left[\left(\frac{1}{\Delta t^{*}}-\frac{3}{2 \Delta t}\right) \mathbf{I}+\frac{\partial \mathbf{R}}{\partial \mathbf{W}}\right] \Delta \mathbf{W}=-\left(\mathbf{R}(\mathbf{W})+\frac{3 \mathbf{W}^{n+1}-4 \mathbf{W}^{n}+\mathbf{W}^{n-1}}{2 \Delta t}\right)
$$

where $\Delta t$ is the real time step. Periodicity can be used to approximate the initial solution for the pseudo time stepping at each real time step. The solution iterates in pseudo time for each real time step to achieve convergence. In the current application, a key functionality of the CFD solver is the ability to move the mesh conforming to the motion of the body. Rigid body motions can be treated by moving the mesh rigidly in response to the applied sinusoidal motion. The mesh is deformed once per real time step during the unsteady calculation. A curvilinear time dependent formulation is used to formulate the mapping between the computational space and the physical space.

\section{B. Cobalt}

The Cobalt code solves the unsteady, three-dimensional and compressible Navier-Stokes equations. The Navier-Stokes equations are discretised on arbitrary grid topologies using a cell-centered finite volume method. Second order accuracy in space is achieved using the exact Reimann solver of Gottlieb and Groth ${ }^{27}$ and least squares gradient calculations using QR factorization. To accelerate the discretized system, a pointimplicit method using analytic first-order inviscid and viscous Jacobian is used. A Newtonian sub-iteration 
method is used to improve time accuracy of the point-implicit method. The method is second order accurate in time. Tomaro et al. ${ }^{28}$ converted the code from explicit to implicit, enabling CFL numbers as high as $10^{6}$. Cobalt uses an arbitrary Lagrangian-Eulerian formulation and hence allows all translational and rotational degrees of freedom. For the control surface simulations, an overset grid capability is available. The code can simulate both free and specified six degrees of freedom motions.

\section{Model Formulations of Unsteady Aerodynamic Loads}

The generality realized in a CFD simulation comes at the expense of computational cost. Routine use of high-fidelity CFD simulations is costly for a variety of applications involving several independent design variables, such as in multidisciplinary optimization, aeroelasticity and flight dynamics studies. The analysis of unsteady flows, in particular, can be a computational challenge due to the time-step size used to accurately simulate the flow dynamics and the duration time of the simulation. This motivates the need to assess the benefits and limitations in using low-order models to predict the resulting unsteady aerodynamic loads.

To accelerate the turn-around time of the investigations, the focus of the current paper is on a symmetric two-dimensional airfoil. While retaining complex flow features due to shock-induced phenomena, the time required for the unsteady time-domain simulations is drastically reduced when compared to three-dimensional cases. It is assumed that any consideration on the readiness of the mathematical models can be transferred to the analysis of more complex geometries.

Low-order mathematical models are used as computationally efficient approximations in place of the non-linear system of equations described in Eq. (2). Nonetheless, the low-order models require sufficient informations to be generated using unsteady time-domain calculations. A consideration is for the cost incurred in the generation of suitable aerodynamic data, and for the selection of appropriate training inputs.

The investigations presented are for the prediction of unsteady aerodynamic loads in response to a harmonic motion about the pitch axis. The angle of attack as function of time is defined as

$$
\alpha(t)=\alpha_{0}+\alpha_{A} \sin (\omega t)
$$

It is also convenient to introduce the non-dimensional reduced frequency of the applied motion, $k=$ $\omega c /\left(2 U_{\infty}\right)$, based on the mean aerodynamic chord and freestream speed. Low-order mathematical models are now formulated.

\section{A. Conventional Model based on Aerodynamic Derivatives}

The concept of stability or aerodynamic derivatives was introduced by Bryan ${ }^{29}$ and defines the conventional model for the representation of the aerodynamic loads in the equations of motion. It is based on the linearization of the aerodynamic terms using the perturbation approach, discarding higher order terms. ${ }^{30}$ Forces and moments are assumed functions of the instantaneous values of the disturbance velocities, control angles and their rates. For slow motions at low angle of attack, static derivatives are typically sufficient to model the aerodynamic loads. The model limitations are exhibited when confronting to flows with non-linear, time-history effects including high-frequency components, which represent flows of practical interest.

Without loss in generality, let consider the longitudinal aerodynamic coefficients, e.g., lift, drag, and pitching moment. The conventional representation of the aerodynamic loads, based on a quasi-steady flow assumption, is

$$
\begin{gathered}
C_{j}=C_{j 0}+C_{j_{\alpha}} \Delta \alpha+C_{j_{\dot{\alpha}}} \frac{c}{2 U_{\infty}} \dot{\alpha}+C_{j_{q}} \frac{c}{2 U_{\infty}} q+C_{j_{\dot{q}}}\left(\frac{c}{2 U_{\infty}}\right)^{2} \dot{q} \\
\quad \text { for } j=L, D \text {, and } m
\end{gathered}
$$

Aerodynamic derivatives are evaluated at a reference condition, e.g., trimmed flight condition, and are assumed to be constant. However, for large amplitude manoeuvres and rapid excursions from the reference flight condition, it is necessary to extend the linear model to include non-linear terms. Following Da Ronch et al., ${ }^{14}$ a non-linear counterpart of Eq. (4) is formulated as

$$
\begin{gathered}
C_{j}=C_{j 0}(\alpha)+C_{j_{\dot{\alpha}}}(\alpha) \frac{c}{2 U_{\infty}} \dot{\alpha}+C_{j_{q}}(\alpha) \frac{c}{2 U_{\infty}} q \\
\text { for } j=L, D, \text { and } m
\end{gathered}
$$


The static term, $C_{j 0}$, depends non-linearly on the angle of incidence, and accounts for static non-linearities in the aerodynamic coefficients. Aerodynamic derivatives, while non-linear functions in the argument, are linear with respect to the angular rates. In addition, when considering a sinusoidal motion about the pitch axis, the above non-linear formulation can be recast in the form of Eq. (6).

$$
\begin{gathered}
C_{j}=C_{j 0}(\alpha, \ldots)+\bar{C}_{j_{q}}(\alpha, \ldots) \frac{c}{2 U_{\infty}} \dot{\alpha} \\
\quad \text { for } j=L, D, \text { and } m
\end{gathered}
$$

The dynamic derivative, $\bar{C}_{j_{q}}=C_{j_{\dot{\alpha}}}+C_{j_{q}}$, includes a rotary derivative and a translation acceleration derivative. Dynamic derivatives are traditionally calculated by post-processing the time-history of the aerodynamic coefficients in response to a sinusoidal motion. Details on the computation of dynamic derivatives can be found in reference. ${ }^{14}$ As described in the mentioned study, the dependence of the dynamic derivatives on motion and flow conditions may be important above the static stall, with a loss in accuracy in the prediction of the unsteady aerodynamic loads. Investigations into computationally efficient methods for predicting dynamic derivatives were presented in reference. ${ }^{16,18}$ In the present study, unsteady time-domain simulations are used to generate the aerodynamic informations for the model represented by Eq. (6). The static non-linear term, $C_{j 0}$, can be obtained either from a static simulation or from an unsteady time-domain simulation as a time-averaged value.

The stability behaviour of a free-to-pitch aircraft model is determined by the sign of the dynamic derivative of the pitching moment coefficient, $\bar{C}_{m_{q}}$, which is generally referred to as the damping-in-pitch. In the dynamic equations of motion governing the free response about the pitch axis, the forcing term on the right hand side is the pitching moment. This term can be moved to the left hand side, beside the inertia term, and the dynamic derivative contribution can be interpreted in analogy with a structural damping term. The free pitching response is stable if and only if the damping-in-pitch term is negative, ${ }^{18}$ or when the equivalent structural damping term is positive.

\section{B. Volterra Series}

The Volterra theory is a well-known subject in non-linear systems, ${ }^{31}$ and it has been applied to systems involving transonic aerodynamics. ${ }^{32}$ The aim of this work is to extend the use of the Volterra theory to the area of stability and control.

Let denote each aerodynamic coefficient as $y=C_{j}$ for $j=L, D, m$. The output of a continuous-time, casual, time-invariant, fading memory system in response to an input, $x(t)$, is formulated as

$$
y(t)=\Psi(x(t))=\sum_{i=1}^{p} \mathbb{H}_{i}(x(t))
$$

The output response, $y(t)$, is modeled using the $p$-th order Volterra series. The term $\mathbb{H}_{i}$ represents the $i$-th order Volterra operator, which is defined as a $i$-fold convolution between the input, $x(t)$, and the $i$-th order Volterra kernel, $H_{i}$.

$$
\mathbb{H}_{i}(x(t))=\int_{-\infty}^{t} \ldots \int_{-\infty}^{t} H_{i}\left(t-\tau_{1}, t-\tau_{2}, \ldots, t-\tau_{i}\right) \prod_{n=1}^{i} x\left(\tau_{n}\right) d \tau_{n}
$$

Based on linear aerodynamic theories, it can be stated that the unsteady aerodynamic loads are functions of the angle of attack and its time derivatives. This conclusion is important in the sense that the single-input Volterra model formulated in Eq. (7) may be inadequate even for the representation of loads in the linear aerodynamic regime. For oscillations about the pitch axis, the relevant external inputs are

$$
\underline{x}(t)=(\alpha(t), \dot{\alpha}(t), \ddot{\alpha}(t))
$$

A multi-input Volterra series is then formulated as

$$
y(t)=\Psi\left(x_{1}(t), x_{2}(t), \ldots, x_{m}(t)\right)=\sum_{i=1}^{p} \mathbb{H}_{i}^{m}
$$


The term $\mathbb{H}_{i}^{m}$ is the multi-input Volterra operator defined as a $m^{p}$-fold summation of $p$-fold convolution integrals between the inputs and the $p$-th order multi-input Volterra kernels. ${ }^{33}$ The output response is rewritten as

$$
\begin{aligned}
y(t)= & \sum_{j=1}^{m} \int_{-\infty}^{t} H_{1}^{x_{j}}(t-\tau) x_{j}(\tau) d \tau+ \\
& \sum_{j_{1}=1}^{m} \sum_{j_{2}=1}^{m} \int_{-\infty}^{t} \int_{-\infty}^{t} H_{2}^{x_{j_{1}}, x_{j_{2}}}\left(t-\tau_{1}, t-\tau_{2}\right) x_{j_{1}}\left(\tau_{1}\right) x_{j_{2}}\left(\tau_{2}\right) d \tau_{1} d \tau_{2}+\ldots
\end{aligned}
$$

Note that the superscripts in Eq. (11) identify to which inputs the kernel corresponds. Let consider, as an example, the second-order kernel $H_{2}^{x_{j_{1}}, x_{j_{3}}}$, which correlates the inputs $x_{j_{1}}$ and $x_{j_{3}}$.

The identification of the Volterra kernels is performed using an unsteady time-domain simulation as source of the data. The CFD solution is discrete in time, and the time-step is indicated by $\Delta t$. Let denoted $x(t)=x(n \Delta t)=x[n]$. The discrete-time representation of Eq. (11) rewrites as

$$
\begin{aligned}
y[n]= & \sum_{j=1}^{m} \cdot \sum_{k=0}^{n} H_{1}^{x_{j}}[n-k] x_{j}[k]+ \\
& \sum_{j_{1}=1}^{m} \sum_{j_{2}=1}^{m} \cdot \sum_{k_{1}=0}^{n} \sum_{k_{2}=0}^{n} H_{2}^{x_{j_{1}}, x_{j_{2}}}\left[n-k_{1}, n-k_{2}\right] x_{j_{1}}\left[k_{1}\right] x_{j_{2}}\left[k_{2}\right]+\ldots
\end{aligned}
$$

The identification of discrete-time Volterra kernels involves the resolution of an overdetermined system. Values of aerodynamic coefficients and the time-history of the motion variables are known from the CFD simulation used as training input. Let $\underline{y}=(y[0], y[1], \ldots, y[n])^{T}$ denote each aerodynamic load computed using CFD, and let $\underline{\underline{A}}$ contain the permutations of input parameters relevant to the unsteady motion. Equation (12) can be recast in the form

$$
\underline{y}=\underline{\underline{A b}}
$$

where the vector $\underline{b}$ contains the unknown Volterra kernels. The matrix $\underline{\underline{A}}$ is in general non-square, with more rows than columns. Several numerical methods are available to solve least squares problems, e.g., direct inversion of $\underline{\underline{A}}^{T} \underline{\underline{A}}$, Gauss elimination, Moore-Penrose generalized inverse approach and the QR factorization. However, the Moore-Penrose approach and the QR factorization are more accurate than the Gauss elimination and the direct inversion solutions. The cost of the QR factorization is $\left[o\left(n^{2}\right)\right]$, and the Moore-Penrose inversion involves $\left[o\left(n^{3}\right)\right]$ operations. Note that computational resources attributable to the identification of the Volterra kernels grow exponentially with order. Increasing the order of the Volterra series introduces a requirement for a training manoeuvre of sufficient duration. A remedy to this is the use of a simplified form of the kernel parametric structure. ${ }^{34}$

$$
\begin{aligned}
& H_{p}^{x_{j_{1}}, x_{j_{2}}, \ldots, x_{j_{p}}}\left[n-k_{1}, n-k_{2}, \ldots, n-k_{p}\right]=0 \\
& \quad \text { for } k_{1} \neq k_{2} \neq \ldots \neq k_{p}
\end{aligned}
$$

The simplified form of the Volterra series is not included in this work because of the non-linear character of the results presented.

Once the kernels are identified, the low-order model formulated in Eq. (13) can be used in place of the full-order system defined in Eq. (2) to predict the unsteady aerodynamic loads for a novel manoeuvre.

\section{Surrogate-Based Recurrence-Framework (SBRF)}

The non-linear system of equations described by Eq. (2) can be interpreted as a general representation of a non-linear time-invariant discrete-time dynamical system. The state vector consists of the conservative variables (density, velocity components, and energy), and its size is proportional to the number of grid points. In this study, the aerodynamic loads form the vector of outputs, which are not only a function of the instantaneous values of the inputs, but also a function of the time history of the inputs.

To generate a computationally efficient approximation of the unsteady aerodynamic loads without solving the expensive equations in Eq. (2), the form of a dynamical system is assumed. ${ }^{35}$ When the state vector of 
the full-order system is finite in number, which is the case of $\mathbf{W}$, the following non-linear system is equivalent to Eq. (2).

$$
y(t)=\Phi(\underline{x}(t), \underline{x}(t-\Delta t), \ldots, \underline{x}(t-m \Delta t), y(t-\Delta t), \ldots, y(t-n \Delta t))
$$

The vector $\underline{x}$ was previously defined in Eq. (9). The function $\Phi$ maps the inputs to the outputs. The terms $m$ and $n$ represent the number of previous values of the external inputs and outputs, respectively, influencing the output at the current time instant. These parameters account for time-history effects and phase-lag in the flow development.

Central to the synthesis of the reduced-order model is the computation of the function $\Phi$. Without a closed-form analytical expression, a numerical approximation of $\Phi$ is constructed using a number of CFD solutions. For the pitching airfoil case represented by Eq. (3), any motion can be expressed as function of three parameters, e.g., $\alpha_{0}, \alpha_{A}$, and $k$. These independent variables form a parameter space, representing the envelope of all possible flow conditions that the airfoil configuration is expected to operate. To generate a consistent set of unsteady aerodynamic loads in response to a given airfoil motion time history, the training cases at which CFD solutions are calculated should be representative of the expected flow conditions. Several design of experiment methods are available in the literature. A description of the Kriging-based framework used in this study is detailed in reference. ${ }^{36}$ Let $N_{T}$ be the number of training cases for which CFD solutions are available. Each training case consists of different combinations of the independent parameters,

$$
\begin{array}{r}
\underline{x}_{i}=\left[\alpha_{i}(t), \dot{\alpha}_{i}(t), \ddot{\alpha}_{i}(t)\right] \\
\text { for } i=1, \ldots, N_{T}
\end{array}
$$

and the corresponding aerodynamic loads are indicated by $y_{i}(t)$. The approximation of the function $\Phi$ is obtained by interpolating the sampled data in the form of input/output relationship. Several interpolation methods are available in the literature, and two of these have been used in the present study. Kriging interpolation is a common choice, but for increasing number of independent parameters the problem can result to be ill-conditioned. An alternative approach is the multi-linear interpolation technique, which is in general faster than the Kriging interpolation.

\section{Indicial Function}

The buildup in the aerodynamic loads in response to a unit step in one of the inputs can be evaluated by convolution. Let $H(t)$ be the unit step, defined as

$$
H(t)= \begin{cases}1.0 & \text { for } \quad t \geq 0 \\ 0.0 & \text { for } \quad t<0\end{cases}
$$

The unit response, or indicial admittance, is denoted by $a(t)$. Assuming a linear relationship between the forcing function and the output, the airloads are defined as the convolution or Durhamel's superposition ${ }^{37}$ of the indicial response with the derivative of the forcing function.

$$
y(t)=\frac{d}{d t} \int_{0}^{t} a(t-\tau) x(\tau) d \tau
$$

This model can approximate any finite-memory, time-invariant, single input/single output and continuous linear system. ${ }^{38}$ The indicial response functions are used as a fundamental approach to represent the unsteady aerodynamic loads. ${ }^{39,40}$ Let $C_{j_{\alpha}}$ and $C_{j_{q}}$ be the time response in the unsteady aerodynamic loads due to a step change in the angle of attack, $\alpha$, and angular velocity, $q$. If these functions are known, then the unsteady airloads at time $t$ can be obtained as

$$
\begin{gathered}
C_{j}(t)=\frac{d}{d t} \int_{0}^{t} C_{j_{\alpha}}(t-\tau) \alpha(\tau) d \tau+\frac{d}{d t} \int_{0}^{t} C_{j_{q}}(t-\tau) q(\tau) d \tau \\
\quad \text { for } j=L, D, \text { and } m
\end{gathered}
$$

These models approximate the unsteady lift and pitching-moment in the linear regimes of flight envelope. For non-linear aerodynamics, the indicial response is computed at several angles of attack. However this adds 
more computational cost for the construction of the model. Linear indicial functions were used in this study. These functions need to be determined prior to the model construction. Note also that a limited number of analytical solutions for the indicial functions over flat-plate in low speed aerodynamics is available in the literature. For compressible and three-dimensional geometries, the only direct method for determination of the impulse and unit response functions is CFD. Experimental testing of step responses is practically non-existent.

\section{E. Radial Basis Function}

In this approach, the unsteady aerodynamic loads are approximated by learning an input-output mapping from a set of training data. ${ }^{41}$ The reconstructed state space model was presented previously in Eq. (15). The term $\Phi$ is a vector-valued nonlinear function that maps the inputs to the output. The terms $m$ and $n$ are integers representing the past values in the output and input, respectively. For ease of programming, the past values of input and output were assumed to be similar, i.e. $m=n$. The mapping function, $\Phi$ is learned through Radial Basis Function Neural Network (RBFNN). The remaining problem is how to choose $n$ such that the reconstructed model accurately represents the state-space model. In this paper the value of $n$ is selected using "trial and error" attempts. Design of experiments are often used to select $N_{T}$ combinations of these variables for training purposes. However, such an approach often needs a large value of $N_{T}$ to cover the important regimes of the regressor space of the input variables. Ghoreyshi et al. ${ }^{41}$ reduced number of manoeuvre simulations using the design of new training maneuvers. This approach was also used in this study.

\section{Results}

The NACA 0012 airfoil is the test case. An inviscid and viscous model were used in the present work. The inviscid grid for use with the PMB solver, illustrated in Fig. 1(a), is structured. The grid consists of 32028 grid points, 212 nodes were distributed on the airfoil, 51 points were used in the normal direction and along the streamwise direction for the wake. The two-dimensional domain extends fifty chords from the solid wall to the farfield. A preliminary study was made to guarantee solutions presented are independent of the grid used. A time-step refinement study was also performed, and further details on the inviscid grid for the PMB solver can be found in reference. ${ }^{18}$ The viscous grid for use with Cobalt has a rectangular computational domain with the airfoil geometry centrally located. The farfield is located twenty chords from the solid wall. The unstructured mesh with prisms in the boundary layer and tetrahedra elsewhere is shown in Fig. 1(b). Note that numerical results were compared with tunnel measurements under static and unsteady conditions, and providing further details on the validation is beyond the scope of the present study.

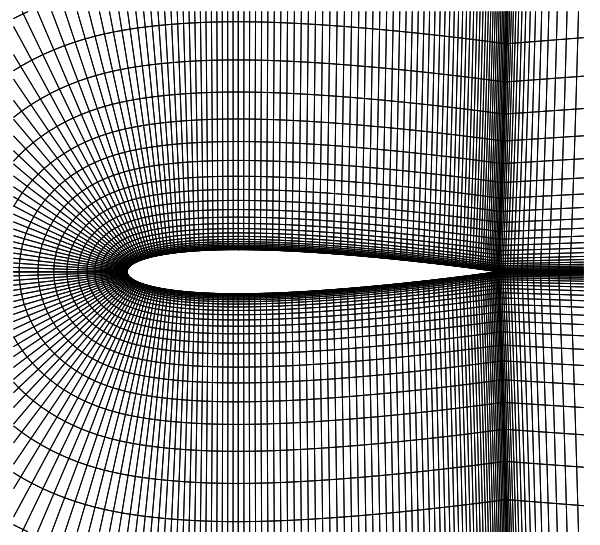

(a) Inviscid grid (PMB)

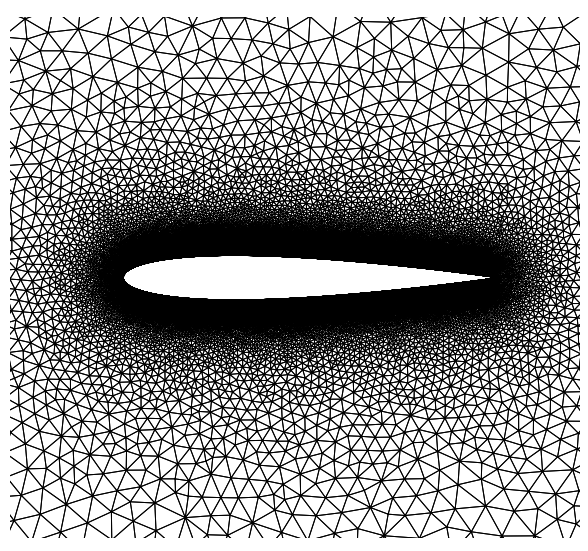

(b) Viscous grid (Cobalt)

Figure 1. Inviscid and viscous grid for the NACA 0012 airfoil 


\section{A. Conventional Model based on Aerodynamic Derivatives}

The experimental conditions of the AGARD CT5 case $^{42}$ feature a harmonic motion about the pitch axis in transonic conditions, at a freestream Mach number of 0.755 . The mean angle of incidence and the oscillatory amplitude are 0.016 and 2.51 degrees, respectively, and the reduced frequency is 0.0814 . Details on the validation of the PMB solver for this case are reported in reference. ${ }^{16,18}$ The flow solution is non-linear, and consists of a shock appearing at the leading-edge and moving downstream for increasing angles. For decreasing angles, the shock moves upstream close to the leading-edge and disappears. The same pattern is observed on the opposite side of the airfoil. The flow remains attached throughout the entire cycle of unsteadiness.

The non-linear model based on the aerodynamic derivatives was used to predict the resulting unsteady aerodynamic loads. Aerodynamic informations for the model represented by Eq. (6) were obtained from a number of small-amplitude sinusoidal oscillations at the same flow conditions of the test case presented. The dynamic derivative of the pitching moment coefficient is illustrated in Fig. 2(a). Data presented were computed from sinusoidal motions for an amplitude of one degree. Note that calculations were repeated for a smaller oscillatory amplitude of 0.5 degrees, with identical aerodynamic informations obtained. The reason for this may be due to the amplitude values considered, and the fact that the flow development for a small oscillatory motion may be linear or quasi-linear. It is considered that deviations in aerodynamic derivatives will be observed when increasing the oscillatory amplitude.

Figure 2(a) conveys the variation of the damping-in-pitch term with the angle of attack. Increasing the mean angle of attack results in a continuous increase in the aerodynamic damping term, and above two degrees this term becomes positive. In the case of free pitching oscillations, the system is dynamically unstable. With computed aerodynamic informations, the non-linear model formulated in Eq. (6) was used for the prediction of the pitching moment dynamic dependence for the AGARD CT5 case. In Fig. 2(b), the unsteady time-domain simulation is referred to as "CFD". The initial transient was removed from the numerical solution, and two arrows indicate the time evolution. If the arrows are oriented in the counterclockwise direction, the contribution from the dynamic derivative is negative, and vice versa. For the sake of clarity, experimental data are not included (see reference ${ }^{16,18}$ for a comparison). The non-linear model is denoted in the same figure by "Model". Deviations between the prediction and the reference solution are observed throughout the entire cycle, and two cross-over points are observed in the model results which are not contained in the reference solution. This suggests that the model of aerodynamic derivatives is incapable of correctly representing the aerodynamic loads for this case, due to significant non-linear features in the flowfield solution. This corroborates the need to consider alternative modeling techniques in cases for which the conventional model based on aerodynamic derivatives exhibits a loss in accuracy.

\section{B. Volterra Series}

The discrete-time multi-input Volterra model formulated in Eq. (12) was used to model the force and moment dynamic dependency for a large-amplitude oscillatory motion in the transonic regime. The Mach number was set to 0.764 and the reduced frequency to 0.10 . The mean angle of attack was zero, and the amplitude of 8.5 degrees was selected to excite non-linear aerodynamics due to shock-induced separation. This testcase is also considered in the next sections.

For the identification of the Volterra kernels, a training manoeuvre of sufficient duration time was generated using CFD as source of the data. The variation of the angle of attack with time is shown in Fig. 3(a). Note that the training input is at the reduced frequency of the manoeuvre to be simulated, and the oscillatory amplitude varies linearly up to fourteen degrees. The dynamic dependence of the pitching moment coefficient computed using CFD is illustrated in Fig. 3(b). The Volterra model is also included in the same figure for comparison with the training signal used in the identification process. The overall agreement is reasonable. However, deviations can be observed at both ends of the time interval.

As the airfoil is symmetric and oscillates around zero degrees mean angle of attack, the resulting aerodynamic loads are odd functions of the angle of attack change. As a result, any odd kernel was neglected in the identification process. The Volterra model used in the results presented includes kernels up to third order as follows

$$
\begin{array}{lll}
H_{1}^{\alpha} & H_{1}^{\dot{\alpha}} & H_{1}^{\ddot{\alpha}} \\
H_{2}^{\alpha, \dot{\alpha}} & H_{2}^{\dot{\alpha}, \ddot{\alpha}} & \\
H_{3}^{\alpha, \dot{\alpha}, \dot{\alpha}} & H_{3}^{\dot{\alpha}, \dot{\alpha}, \ddot{\alpha}} & H_{3}^{\alpha, \alpha, \ddot{\alpha}}
\end{array}
$$




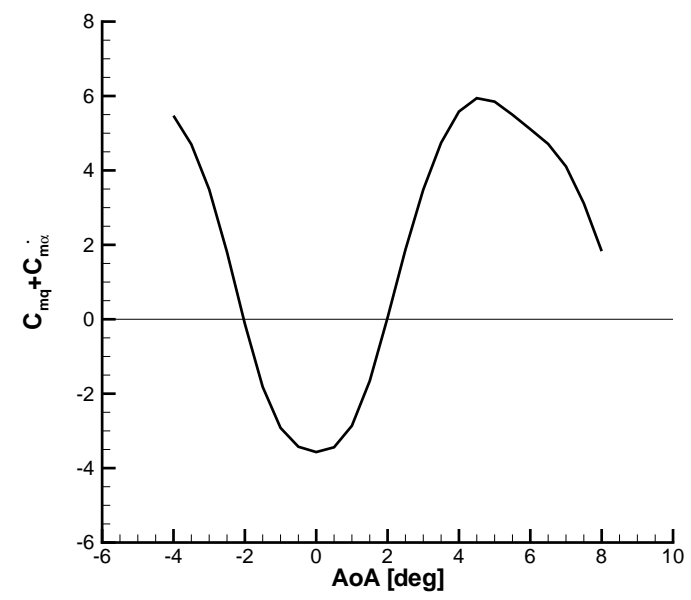

(a) Damping-in-pitch

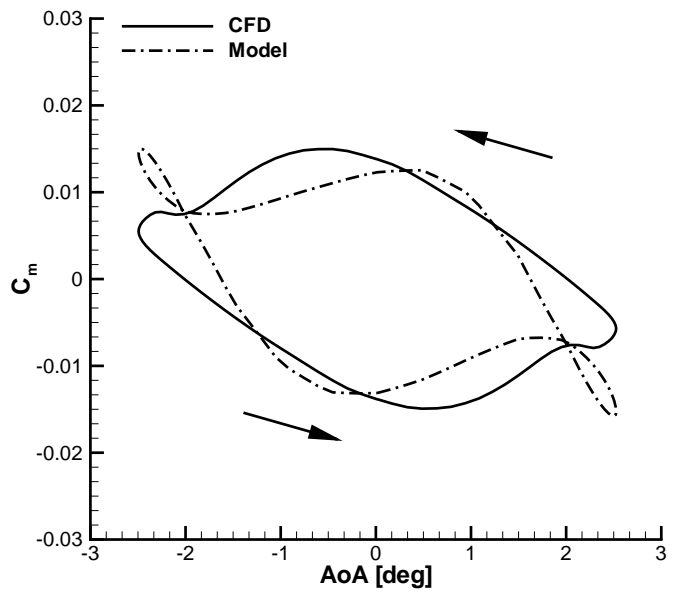

(b) Pitching moment coefficient

Figure 2. NACA 0012: predictions of pitching moment dynamic dependence for the AGARD CT 5 case $\left(M=0.755, \alpha_{0}=0.016^{\circ}, \alpha_{A}=2.51^{\circ}\right.$, and $\left.k=0.0814\right)$; in (a), dynamic derivative of the pitching moment; in (b), "Model" refers to the non-linear model based on aerodynamic derivatives

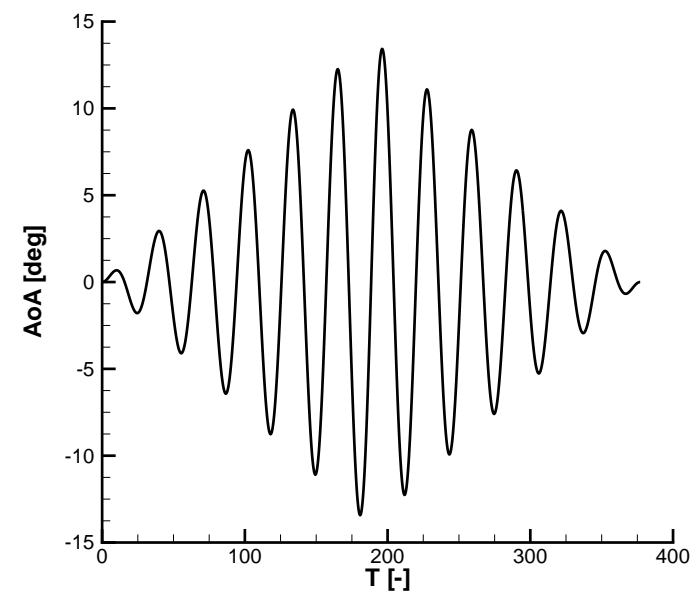

(a) Angle of attack

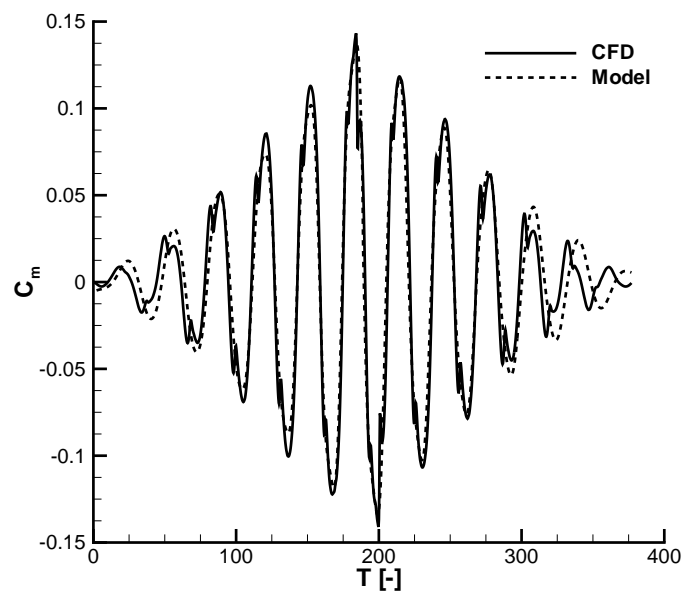

(b) Pitching moment coefficient

Figure 3. NACA 0012: training manoeuvre for the generation of a discrete-time multi-input Volterra model $\left(M=0.764, \alpha_{0}=0.0^{\circ}\right.$ and $\left.k=0.10\right)$; in (a), variation of angle of attack with time; in (b), "Model" refers to the discrete-time multi-input Volterra model 
The upper bound of the integer $k$ in Eq. (12), which indicates the number of previous time-steps to account for, was set to 1 for the terms $H_{1}^{\alpha}$ and $H_{1}^{\dot{\alpha}}$. For the other kernels, it was set to 0 .

The effect of including more terms in the model was assessed during the identification of the Volterra model, and in the subsequent comparison for the novel manoeuvre. The error norm was defined as

$$
E=\frac{\sum_{i=0}^{N}\left|y^{\mathrm{cfd}}[i]-y^{\mathrm{rom}}[i]\right|}{y_{\max }^{\mathrm{cfd}}-y_{\min }^{\mathrm{cfd}}} \cdot 100
$$

where $N$ is the total number of time-steps used in the CFD simulation, and the superscripts cfd and rom indicate, respectively, the full-order and the reduced-order model. Including first order kernels, the error in the prediction of the large-amplitude manoeuvre was $6.78 \%$. Second order kernels were then added to the first order kernels, and the Volterra model deviated by $6.87 \%$ from the CFD solution. By introducing third order kernels, the error norm slightly decreased to $6.18 \%$.

Figure 4 conveys the unsteady pitching moment coefficient for the large-amplitude manoeuvre. The CFD solution exhibits non-linear characteristics at the higher angles of attack. The Volterra model including the kernels shown in Eq. (20) is illustrated in the same figure for comparison. Despite using kernels up to third order, the agreement is not excellent. The overall fit of the model is reasonable, but there is no sign of the prediction of any non-linear feature.

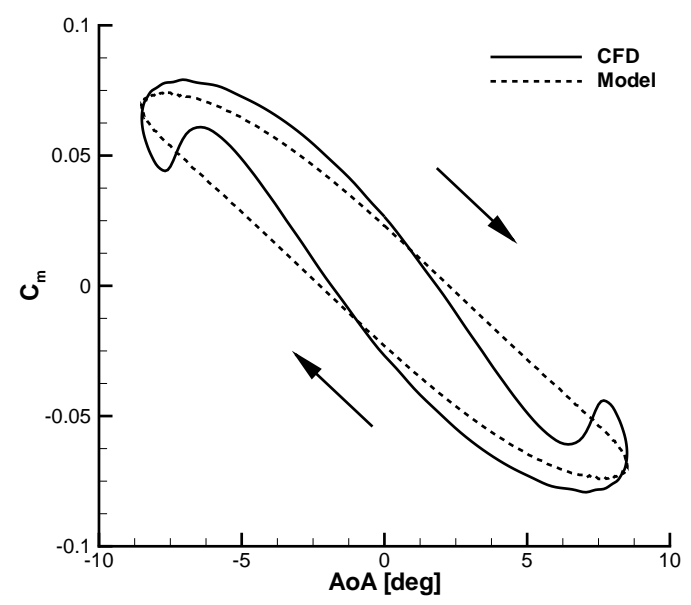

Figure 4. NACA 0012: predictions of pitching moment dynamic dependence $\left(M=0.764, \alpha_{0}=0.0^{\circ}, \alpha_{A}=8.5^{\circ}\right.$, and $k=0.10)$; "Model" refers to the discrete-time multi-input Volterra model

The mediocre performance of the Volterra model may be attributable to two aspects. To obtain a robust enough predictive model, in combination with kernels of the second and third order, the influence of the previous time-history on the current time instant was limited. Second, the training case considered may be not optimal for the model identification. In general, a suitable training manoeuvre is designed to allow sufficient representative data to create a low-order model with predictive capabilities within a desired parameter space. Previous research focused on training manoeuvres development, ${ }^{43,44}$ but it is argued that an appropriate manoeuvre used to predict stability and control characteristics is not primarily given by its frequency content or power density spectra. ${ }^{45}$ As the low-order model is used to predict the aerodynamic behaviour within certain flight and control states, a sufficient coverage of the parameter space should be realized by the design manoeuvre to be effective. The downside of this consideration is the likely nonphysical nature of the training inputs used for the Volterra model, constraining the model primarily to numerical simulations.

\section{Surrogate-Based Recurrence-Framework}

The results presented in this section correspond to modeling the unsteady aerodynamic loads for the large amplitude manoeuvre considered for the Volterra model. The Mach number is 0.764 , the reduced frequency 
is 0.10 , and the amplitude is 8.5 degrees for sinusoidal oscillations around zero degrees mean angle of attack.

The SBRF model was constructed from CFD solutions corresponding to 30 different combinations of $\alpha_{A}$, constrained to vary between $0.0^{\circ}$ and $10.0^{\circ}$. With an increment in $\alpha_{A}$ of $0.25^{\circ}$, the parameter space was initially covered for $75 \%$ of all the possible combinations. Figure 6 depicts the carpet plot of the pitching moment coefficient for each value of the parameter $\alpha_{A}$ obtained using the SBRF model. The axis "Iteration" indicates the time evolution through the last cycle of the simulations.

Next, the number of initial training CFD solutions was iteratively reduced to focus on the predictive capabilities of the model. Four CFD solutions were eventually retained, and these are included as large spheres in Fig. 6. Note that two training cases are located at the borders of the parameter space to avoid extrapolation. The remaining two cases were automatically sampled by the algorithm described in reference. ${ }^{36}$ Note also that there is no close proximity of the four training cases to the amplitude of 8.5 degrees.

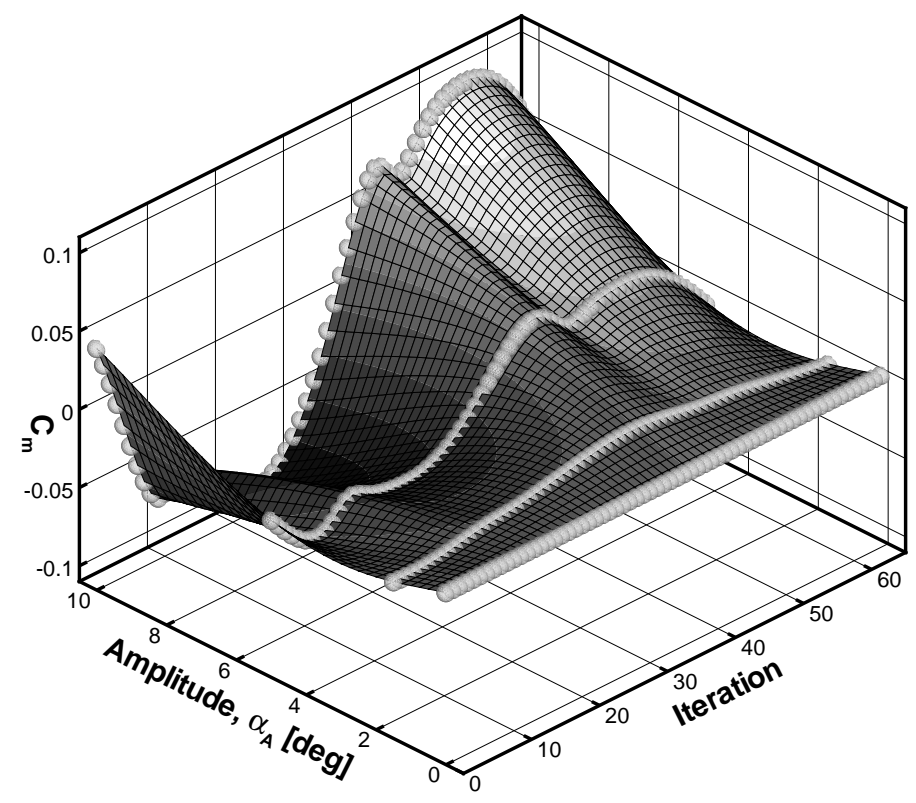

Figure 5. NACA 0012: carpet plot of the pitching moment coefficient $\left(M=0.764, \alpha_{0}=0.0^{\circ}\right.$, and $\left.k=0.10\right)$; large spheres indicate the 4 CFD solutions used to construct the SBRF model

For the results presented in Fig. 6, the SBRF model was constructed from four CFD solutions illustrated in Fig. 6. The reduced-order model closely approximate the reference solution, featuring the very similar non-linear characteristics at higher angles. Little or no sensitivity was found for the value of $m$, introduced in Eq. (15), while the value of $n$ had a larger impact on the predictions. The values used in this study are $m=2$ and $n=1$.

\section{Indicial Function and Radial Basis Function}

The indicial responses of the airfoil with a unit step change of angle of the attack and pitch rate are shown in Fig. 7. The figure shows the responses per radian as a function of non-dimensional time. The lift responses have an initial peak followed by a falling trend. This can be explained based on the energy of acoustic wave system created by the initial perturbation. ${ }^{37}$ The initial peak becomes smaller as the Mach number increases. It was found that translating the grid (angle of attack response), has larger effects on the lift changes compared with rotating motions. Note that the pitching moment has a negative peak as the grid starts to move. The computational cost for each response is around the cost of 10 steady-state calculations.

The design of a new manoeuvre allows to reduce the number of training motions. The training manoeuvre used in this study is a spiral maneuver which consists of a sweep in the amplitude, described as

$$
\alpha(t)=\alpha_{A} t \sin (\omega t)
$$




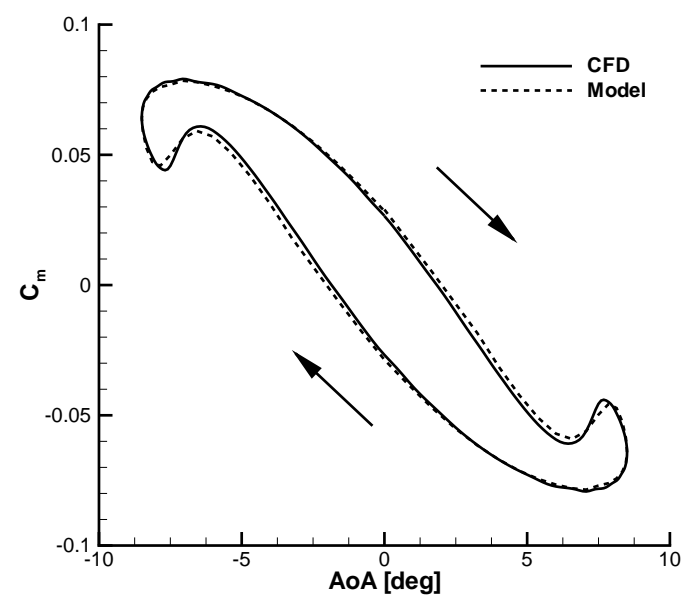

Figure 6. NACA 0012: predictions of pitching moment dynamic dependence $\left(M=0.764, \alpha_{0}=0.0^{\circ}, \alpha_{A}=8.5^{\circ}\right.$, and $k=0.10)$; "Model" refers to the surrogate-based recurrence-framework

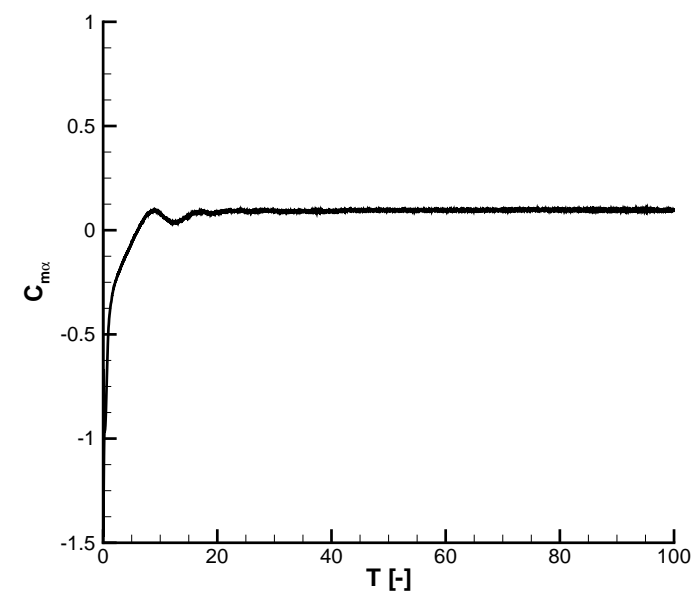

(a) Step change in angle of attack

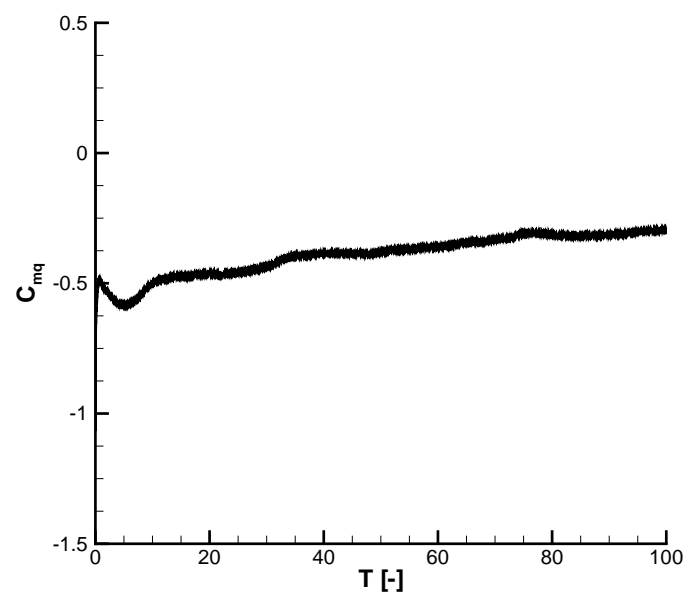

(b) Step change in pitch rate

Figure 7. NACA 0012: indicial responses of pitching moment coefficient to step change in angle of attack and pitch rate $\left(M=0.764\right.$ and $\left.R e=3.0 \cdot 10^{6}\right)$ 
The spiral motion eliminates the need of repeating motions for different values of amplitude. However, for reduced frequency effects, the motion with different values of $\omega$ need to be considered. In this study a spiral motion was defined starting from zero degrees angle of attack at reduced frequency of 0.10 . The simulation was run for 35 oscillatory cycles, which is about 40 times the cost of a single steady-state solution. As in the case of Fig. 3(b), the aerodynamic loads are non-linear with respect to angle of attack change. For RBFNN training, the number of calculated data was reduced on the order of thousands using an interpolation scheme. This allows a faster network training and avoids any out-of-memory error. The reduced spiral data were rearranged according to Eq. (15) and then the network performance was tested for different values of $n$. The results showed that using $n=2$ and $n=4$ is sufficient for modeling the lift and the pitching moment, respectively.

Low-order models based on indicial functions were created using Eq. (15). Also, the networks were trained using training data in the form of Eq. (15). The validity of these models is now tested for several motions. For a pitch oscillation in the linear regime, the results of the reduced order models compared well with the unsteady time-domain solution. This is not unexpected because for a small amplitude, e.g., one degree, the aerodynamics is mostly linear. Figure 8 conveys the non-linear results for the large amplitude pitch oscillation. The results show that indicial functions is off since the model formulation is valid only in linear regimes. On the other hand, the RBFNN predicts the overall trend of the pitching moment.

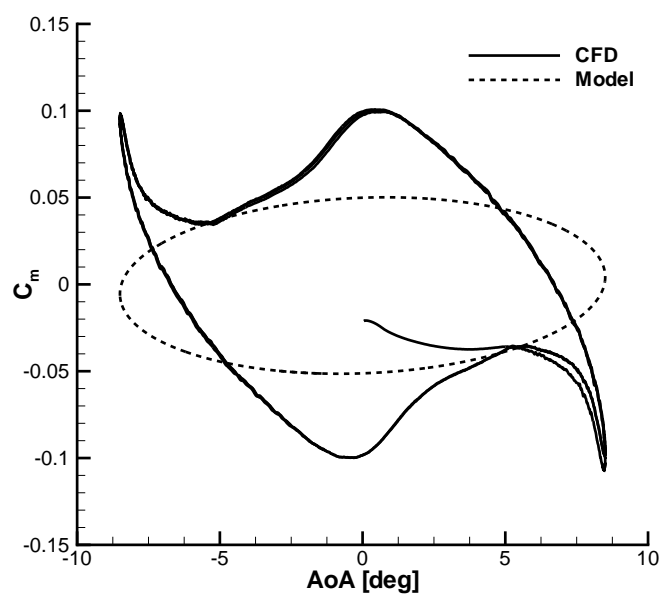

(a) Linear indicial functions

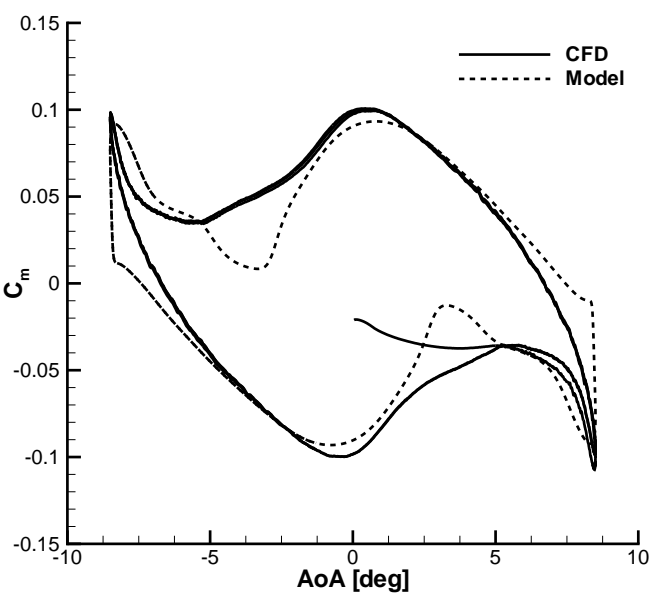

(b) Radial basis functions

Figure 8. NACA 0012: predictions of pitching moment dynamic dependence $\left(M=0.764, \alpha_{0}=0.0^{\circ}, \alpha_{A}=8.5^{\circ}\right.$, $k=0.10$, and and $\left.R e=3.0 \cdot 10^{6}\right)$; in (a), "Model" refers to the linear indicial functions, and in (b) to radial basis functions

\section{Conclusions}

Previous works of the authors demonstrated that the traditional model formulation of aerodynamic loads based on aerodynamic derivatives may experience a loss in accuracy for flow conditions of practical interest. The present work reflects the demand for exploring alternative mathematical formulations for the prediction of unsteady airlods. The testcase is the NACA 0012 airfoil to allow a fast turn-around time of the investigations. The purpose of considering a pitching airfoil at transonic conditions is to establish the effectiveness of the reduced-order models in a non-linear regime of the flow envelope. The non-linearities are attribuitable to shock-induced separation.

Several modeling formulations were presented. A non-linear model based on aerodynamic derivatives, a multi-input discrete-time Volterra model, a surrogate-based recurrence-framework model, linear indicial functions and radial basis functions trained with neural networks were considered. To assess the predictions, low-order models were compared to unsteady time-domain Computational Fluid-Dynamics (CFD) simulations for oscillatory pitching motions.The conventional model based on aerodynamic derivatives exhibited 
large deviations from the reference solution. While retaining higher order Volterra kernels, the multi-input discrete-time model achieved a mediocre agreement, not pinpointing any non-linear feature in the aerodynamic loads. The use of linear indicial functions demonstrated inadequate for the conditions considered. The remaining two models, e.g., surrogate-based recurrence-framework and radial basis functions, achieved a good agreement with the CFD solution. In particular, these models were generated at a cost similar to the cost of computing the aerodynamic information for the conventional model of aerodynamic derivatives.

\section{Acknowledgments}

The first author wishes to thank Maciej Balajewicz of Duke University for an invaluable discussion on the Volterra formulation. McCracken was supported by an Airbus PhD studentship. Ghoreyshi gratefully acknowledges the financial support by the National Research Council/US Air Force Office of Scientific Research.

\section{References}

${ }^{1}$ Chambers, J. R. and Hall, R. M., "Historical Review of Uncommanded Lateral-Directional Motions at Transonic Conditions," Journal of Aircraft, Vol. 41, No. 3, 2004, pp. 436-447.

${ }^{2}$ Woodson, S. H., Green, B. E., Chung, J. J., Grove, D. V., Parikh, P. C., and Forsythe, J. R., "Understanding Abrupt Wing Stall with Computational Fluid Dynamics," Journal of Aircraft, Vol. 42, No. 3, 2005, pp. 578-585.

${ }^{3}$ Forsythe, J. R., Fremaux, C. M., and Hall, R. M., "Calculation of Static and Dynamic Stability Derivatives of the F/A18E in Abrupt Wing Stall Using RANS and DES," Computational Fluid Dynamics 2004, edited by C. Groth and D. W. Zingg, Springer Berlin Heidelberg, 2006, pp. 537-542, doi: 10.1007/3-540-31801-1_76.

${ }^{4}$ Kimberlin, R. D., Flight Testing of Fixed-Wing Aircraft, AIAA Educational Series, Reston, Virginia, USA, 2003. 1999.

${ }^{5}$ Barlow, J. B., William, H. R., and Pope, A., Low-Speed Wind Tunnel Testing, Third Edition, John Wiley \& Sons, Inc.,

${ }^{6}$ Williams, J. E. and Vukelich, S. R., "The USAF Stability and Control Digital DATCOM," McDonnell Douglas Astona UTICS Company, St Louis Division, St Louis, Missouri, 63166, 1979, AFFDL-TR-79-3032.

${ }^{7}$ Boelens, O. J., Badcock, K. J., Elmilgui, A., Abdol-Hamid, K. S., and Massay, S. J., "Comparison of Measured and Block Structured Simulation Results for the F-16XL Aircraft," Journal of Aircraft, Vol. 46, No. 2, 2009, pp. 377-384.

${ }^{8}$ Rogers, S. E., Aftomis, M. J., Pandya, S. A., Chaderjian, N. M., Tejnil, E. T., and Ahmad, J. U., "Automated CFD Parameter Studies on Distributed Parallel Computers," AIAA Paper 2003-4229, 2003.

${ }^{9}$ Ghoreyshi, M., Badcock, K. J., and Woodgate, M. A., "Accelerating the Numerical Generation of Aerodynamic Models for Flight Simulation," Journal of Aircraft, Vol. 46, No. 3, 2009, pp. 972-980.

${ }^{10}$ Ghoreyshi, M., Da Ronch, A., Badcock, K. J., Dees, J., Bérard, A., and Rizzi, A., "Aerodynamic Modelling for Flight Dynamics Analysis of Conceptual Aircraft Designs," 27th AIAA Applied Aerodynamics Conference, AIAA-2009-4121, San Antonio, TX, 2009.

${ }^{11}$ Da Ronch, A., McFarlane, C., Beaverstock, C., Oppelstrup, J., Zhang, M., and Rizzi, A., "Benchmarking CEASIOM Software to Predict Flight Control and Flying Qualities of the B-747," Proceedings of the 27th Congress of the International Council of the Aeronautical Sciences, ICAS 2010-5.10.1, Nice, France, 2010.

${ }^{12}$ McFarlane, C., Richardson, T. S., Da Ronch, A., and Badcock, K. J., "Comparison of Conventional and Asymmetric Aircraft Configurations Using CEASIOM," AIAA Atmospheric Flight Mechanics Conference, AIAA-2010-8243, Toronto, Ontario, 2010.

${ }^{13}$ Da Ronch, A., Vallespin, D., Ghoreyshi, M., and Badcock, K. J., "Computation of Dynamic Derivatives Using CFD," 28th AIA A Applied Aerodynamics Conference, AIAA-2010-4817, Chicago, IL, 2010.

${ }^{14}$ Da Ronch, A., Vallespin, D., Ghoreyshi, M., and Badcock, K. J., "Evaluation of Dynamic Derivatives Using Computational Fluid Dynamics," To appear in AIAA Journal, 2011.

${ }^{15}$ Woodgate, M. and Badcock, K., "Implicit Harmonic Balance Solver for Forced Motion Transonic Flow," AIAA Journal, Vol. 47, No. 4, 2008, pp. 893-901.

${ }^{16}$ Da Ronch, A., Ghoreyshi, M., Badcock, K. J., Görtz, S., Widhalm, M., Dwight, R. P., and Campobasso, M. S., "Linear Frequency Domain and Harmonic Balance Predictions of Dynamic Derivatives," 28th AIAA Applied Aerodynamic Conference, AIAA-2010-4699, Chicago, IL, 2010.

${ }^{17}$ Mialon, B., Khrabrov, A., Da Ronch, A., Cavagna, L., Mengmeng, Z., and Ricci, S., "Benchmarking the Prediction of Dynamic Derivatives: Wind Tunnel Tests, Validation, Acceleration Methods," AIAA Atmospheric Flight Mechanics Conference, AIAA-2010-8244, Toronto, Ontario, 2010.

${ }^{18}$ Da Ronch, A., McCracken, A., Badcock, K. J., Widhalm, M., and Campobasso, M. S., "Linear Frequency Domain and Harmonic Balance Predictions of Dynamic Derivatives," Submitted to International Journal for Numerical Methods in Fluids, 2011.

${ }^{19}$ Ghoreyshi, M., Badcock, K. J., Da Ronch, A. Marques, S., Swift, A., and Ames, N., "Framework for Estabilishing the Limits of Tabular Aerodynamic Models for Flight Dynamics Analysis," Presented as AIAA-2009-4121, Under Review for Publication in Journal of Aircraft.

${ }^{20}$ Ghoreyshi, M., Vallespin, D., Badcock, K. J., Da Ronch, A., Vos, J., and Hitzel, S., "Flight Manoeuvre Validation of 
Data Tables Generated Using an Aerodynamic Model Hierarchy," AIAA Atmospheric Flight Mechanics Conference, AIAA2010-8239, Toronto, Ontario, 2010.

${ }^{21}$ Schiavetta, L. A., Boelens, O. J., Crippa, S., Cummings, R. M., Fritz, W., and Badcock, K. J., "Shock Effects on Delta Wing Vortex Breakdown," Journal of Aircraft, Vol. 46, No. 3, 2009, pp. 903-914.

${ }^{22}$ Vallespin, D., Da Ronch, A., Boelens, O., and Badcock, K. J., "Validation of Vortical Flow Predictions for a UCAV Wind Tunnel Model," 28th AIAA Applied Aerodynamic Conference, AIAA-2010-4560, 2010.

${ }^{23}$ Badcock, K. J., Richards, B. E., and Woodgate, M. A., "Elements of Computational Fluid Dynamics on Block Structured Grids using Implicit Solvers," Progress in Aerospace Sciences, Vol. 36, 2000, pp. 351-392.

${ }^{24}$ Osher, S. and Chakravarthy, S., "Upwind Schemes and Boundary Conditions with Applications to Euler Equations in General Geometries," Journal of Computational Physics, Vol. 50, No. 3, 1983, pp. 447-481.

${ }^{25}$ Van Leer, B., "Towards the Ultimate Conservative Difference Scheme II: Monotonicity and Conservation Combined in a Second Order Scheme," Journal of Computational Physics, Vol. 14, No. 4, 1974, pp. 361-374.

${ }^{26}$ Jameson, A., "Time Dependent Calculations Using Multigrid, with Applications to unsteady Flows Past Airfoils and Wings," AIAA Paper 91-1596, 1991.

${ }^{27}$ Gottlieb, J. J. and Groth, C. P. T., "Assessment of Riemann Solvers For Unsteady One-dimensional Inviscid Flows of Perfect Gasses," Journal of Computational Physics, Vol. 78, No. 2, 1988, pp. 437-458.

${ }^{28}$ Tomaro, R. F., Strang, W. Z., and Sankar, L. N., "An Implicit Algorithm For Solving Time Dependent Flows on Unstructured Grids," AIAA Paper 1997-0333, 1997.

${ }^{29}$ Bryan, G. H., "Stability in Aviation," MacMillan, London, 1911.

${ }^{30}$ Etkin, B., "Dynamics of Atmospheric Flight. Stability and Control," John Wiley \& Sons, New York, 1972. 1981.

${ }^{31}$ Rugh, W. J., Nonlinear System Theory. The Volterra/Wiener Approach, John Hopkins Univ. Press, Baltimore, MD,

${ }^{32}$ Silva, W. A., "Application of Nonlinear Systems Theory to Transonic Unsteady Aerodynamics Responses," Journal of Aircraft, Vol. 30, No. 5, 1993, pp. 660-668.

${ }^{33}$ Balajewicz, M., Nitzsche, F., and Feszty, D., "Application of Multi-Input Volterra Theory to Nonlinear Multi-Degree-ofFreedom Aerodynamic Systems," AIAA Journal, Vol. 48, No. 1, 2010, pp. 56-62, doi: 10.2514/1.38964.

${ }^{34}$ Balajewicz, M., Nitzsche, F., and Feszty, D., "Reduced Order Modeling of Nonlinear Transonic Aerodynamics Using a Pruned Volterra Series," 50th AIAA/ASME/ASCE/AHS/ASC Structures, Structural Dynamics and Materials Conference, Palm Springs, CA 2009.

${ }^{35}$ Glaz, B., Liu, L., and Friedmann, P. P., "Reduced-Order Nonlinear Unsteady Aerodynamic Modeling Using a SurrogateBased Recurrence Framework," AIAA Journal, Vol. 48, No. 10, 2010, pp. 2418-2429, doi: 10.2514/1.J050471.

${ }^{36}$ Da Ronch, A., Ghoreyshi, M., and Badcock, K. J., "On the Generation of Flight Dynamics Aerodynamic Tables by Computational Fluid Dynamics," Submitted for publication in Progress in Aerospace Sciences, 2011.

${ }^{37}$ Leishman, J., "Indicial Lift Approximations for Two-Dimensional Subsonic Flow as Obtained from Oscillatory Measurements," Journal of Aircraft, Vol. 30, No. 3, 1993, pp. 340-351.

${ }^{38}$ Ghoryeshi, M., Jirasek, A., Post, M. L., Cummings, R. M., and Decker, R., "A Computational Investigation into the Use of Response Functions for Aerodynamic Loads Modeling," AIAA Paper 2011-3518, 2011.

${ }^{39}$ Tobak, M., Chapman, G. T., and Schiff, L. B., "Mathematical Modeling of the Aerodynamic Characteristics in Flight Dynamics," NASA TM-85880, 1984.

${ }^{40}$ Tobak, M. and Chapman, G. T., "Nonlinear Problems in Flight Dynamics Involving Aaerodynamic Bifurcations," NASA TM-86706, 1985.

${ }^{41}$ Ghoryeshi, M., Jirasek, A., Post, M. L., and Cummings, R. M., "Computational Approximation of Nonlinear Aerodynamics Using and Aerodynamic Model Hierachy," AIAA Paper 2011-3667, 2011.

${ }^{42}$ Landon, R. H., "NACA 0012. Oscillating and Transient Pitching," Compendium of Unsteady Aerodynamic Measurements, Data Set 3, AGARD-R-702, August 1982.

${ }^{43}$ O'Neill, C. R. and Arena Jr., A. S., "Time-Domain Training Signals Comparison for Computational Fluid Dynamics Based Aerodynamic Identification," Journal of Aircraft, Vol. 42, No. 2, 2005, pp. 421-428.

${ }^{44}$ Jirasek, A. and Cummings, R. M., "Application of Volterra Functions to X-31 Aircraft Model Motion," Collection of Technical Papers - AIAA Applied Aerodynamics Conference, San Antonio, TX, 2009.

${ }^{45}$ Jirasek, A., Jeans, T. L., Martenson, M., Cummings, R. M., and Bergeron, K., "Improved Methodologies for Maneuver Design of Aircraft Stability and Control Simulations," 48th AIAA Aerospace Sciences Meeting Including the New Horizons Forum and Aerospace Exposition, Orlando, FL, 2010. 